\title{
Bottomonia Suppression from AdS/CFT
}

\section{N. N. Barnard}

Department of Physics, University of Cape Town, Private Bag X3, Rondebosch 7701, South Africa

E-mail: brnnad007@myuct.ac.za

\section{W. A. Horowitz*}

Department of Physics, University of Cape Town, Private Bag X3, Rondebosch 7701, South Africa

E-mail: wa.horowitz@uct.ac.za

We compute for the first time the suppression of bottomonia in a strongly-coupled QGP and compare the results to those from a weakly-coupled QGP. Using imaginary time techniques we numerically determine the real and imaginary parts of the ground state binding energy of the bottomonia in one potential computed from AdS/CFT and another computed from pQCD. We then use these binding energies in a suppression model to determine the $\Upsilon(1 \mathrm{~S})$ nuclear modification factor in $\sqrt{s}_{N N}=2.76 \mathrm{TeV} \mathrm{Pb}+\mathrm{Pb}$ collisions. AdS/CFT significantly overpredicts the suppression compared to data, although the predictive power of our calculation is limited by its significant sensitivity to the exact details of the suppression model, especially its treatment of the underlying geometry.

International Conference on Hard and Electromagnetic Probes of High-Energy Nuclear Collisions 30 September - 5 October 2018

Aix-Les-Bains, Savoie, France

\footnotetext{
* Speaker.
} 


\section{Introduction}

Matsui and Satz [1] were the first to propose that suppression of the $J / \psi$ meson spectrum should be observed in the QGP due to Debye-screening of the color charge. Also pioneered in [1] was the use of potential models to describe the interaction of the quark and antiquark in the $q \bar{q}$ pair to calculate the suppression of quarkonia spectra in heavy-ion collisions. In these potential models, the large mass and small relative velocity of the heavy quarks justifies the use of non-relativistic quantum mechanics to describe the quarkonia: the non-relativistic Schrödinger equation gives a binding energy for the quarkonia given a model potential for the $q \bar{q}$ interaction. Further works $[2,3]$ have shown that in addition to a standard real Debye-screened term, the potential of heavy quarkonia at finite temperature contains an imaginary part which gives the thermal width of the state, and hence its suppression. The potential for static heavy quarkonia at finite temperature in $\mathscr{N}=4$ super Yang-Mills (SYM) theory was calculated via the methods of AdS/CFT by [4, 5, 6]. Liu, Rajagopal and Wiedemann (LRW) [7] were the first to present a description from AdS/CFT of the consequences of velocity on the screening length of charmonium. They found that the plasma screening length decreased with velocity and therefore could result in a significant additional source of suppression at high transverse momentum $p_{T}$. We would ultimately like to investigate the consequences of these different quarkonia pictures from AdS/CFT compared to pQCD, to add to the list of observables with both weak- and strong-coupling comparisons $[8,9$, 10]. In this proceedings we have a more modest goal: to compare the experimentally measurable consequences of $\mathrm{pQCD}$ vs. AdS/CFT pictures for computing the quarkonia potential when the quarkonia are at rest with respect to the medium.

One of the challenges of quarkonia research, however, is the significant number of unknowns that cloud the interpretation of $R_{A A}$. For example, even in $p+p$ collisions, the production mechanism for quarkonia is not under good theoretical control [11, 12]. By focusing on bottomonia, the bound state of two bottom quarks, each of mass $m_{b}$, whose formation time $\sim 1 / 2 m_{b} \ll \tau_{f o r m}$, where $\tau_{\text {form }}$ is the formation time of the QGP medium in $A+A$ collisions, we hope to limit our theoretical uncertainty due to quarkonia formation physics. Another complication is the possibility for regeneration. Since $m_{b} \gg T$ at RHIC and LHC and the hard production cross section for bottom is small enough, we can avoid considering regeneration in our bottomonia calculations [13].

\section{Results}

For the details of the heavy quark potentials, the specifics of the numerics for computing the binding energies, and for the suppression model, see [15]. Fig. 1a is a plot of the real part of the binding energy of the ground state of bottomonia, $\Upsilon(1 S)$, from the pQCD potential and stronglycoupled potential as a function of temperature. Similarly, Fig. 1b gives the imaginary part of the binding energies for all cases mentioned above. For the AdS/CFT results, we show the binding energy both for the case where the 't Hooft coupling constant is $\lambda=10$ (labeled as $\alpha_{s}=0.27$, where $\alpha_{s}$ is to be understood as the coupling constant for QCD) and where $\lambda=5.5$ [16]. The binding energy results for bottomonium from [14] are labeled "pQCD (KRS)" and are included for comparison. Both the binding energy results presented for the PQCD potential and the AdS/CFT potential taking $\lambda=10$ were independently confirmed using a complex variational method. The binding 


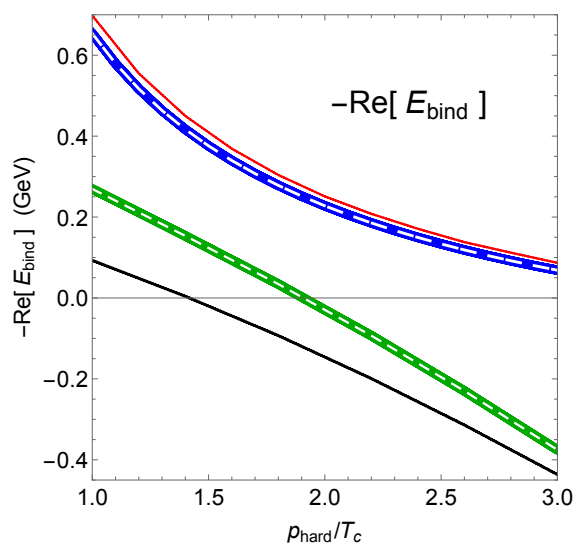

(a)

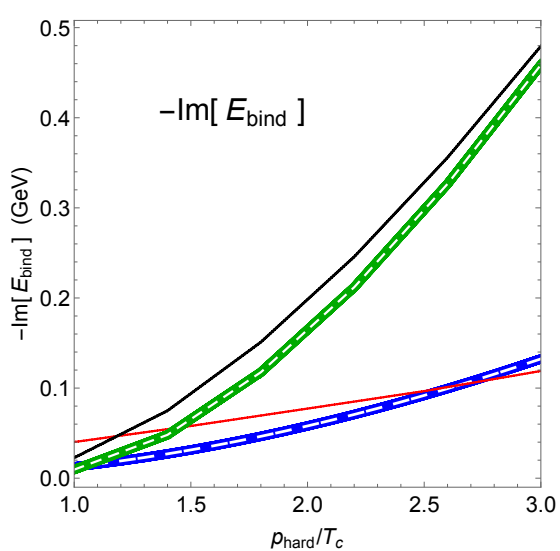

(b)

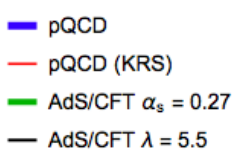

Figure 1: The (a) negative real part of $E_{\text {bind }}$ and (b) negative imaginary part of $E_{\text {bind }}$ for $\Upsilon(1 S)$. The blue, green, and black curves give the results for weakly-coupled and strongly-coupled $(\lambda=10$ and $\lambda=5.5)$ $\Upsilon(1 \mathrm{~S})$, respectively, computed from the imaginary time method. The dashed white curves inside the blue and green curves are from the independent evaluation using the complex variational method. The results from KRS [14], which should be identical to the blue curves, are given as solid red for comparison.

energy found for the pQCD potential differs quantitatively from that presented in [14], which was used in Krouppa et al. [13] to calculate suppression. In the case of $\Upsilon(1 \mathrm{~S})$, this difference does not change the qualitative behavior of the quarkonia, since both results suggest that the quarkonia remain bound up to at least $T=3 T_{c}$, where $T_{c}=192 \mathrm{MeV}$ is the phase transition temperature [14]. However, we will see that the small quantitative differences in the derived binding energies lead to a significant quantitative difference in the predicted suppression. The imaginary part of the binding energies from AdS/CFT are notably larger than those of weakly-coupled quarkonia, and rise more steeply. This result is not surprising as the AdS/CFT potential has a divergent imaginary part, compared to the saturation of the imaginary part of the PQCD potential. Unlike in the case of the weakly-coupled quarkonia where the $\Upsilon(1 \mathrm{~S})$ remains bound for the temperature range considered, the strongly-coupled $\Upsilon(1 \mathrm{~S})$ dissociates at $\sim 1.9 T_{c}$. The comparatively larger imaginary part of the binding energy up to the temperature at which the bottomonium dissociates implies a much larger thermal width at higher $T$, and hence a larger suppression.

Fig. 2a gives the nuclear modification factor $R_{A A}$ for each of the sets of binding energies shown in Fig. 1a and $1 \mathrm{~b}$ as a function of the number of participating nucleons $N_{\text {part }}$. To this end, the $R_{A A}\left(p_{T}, b\right)$ is averaged over the transverse momentum range $0 \leq p_{T} \leq 40 \mathrm{GeV}$ with a weighting of $E^{-4}$ [13]. Fig. 2b shows $R_{A A}\left(p_{T}\right)$, where all centrality classes are included, weighted by the number of binary nucleon-nucleon collisions $N_{\text {coll }}$. Suppression results for mid-rapidity $(|y|<2.4)$ $\mathrm{Pb}+\mathrm{Pb}$ collisions at $\sqrt{s_{\mathrm{NN}}}=2.76 \mathrm{TeV}$ from the CMS Collaboration [17] are included in Fig. 2a and $2 \mathrm{~b}$ for comparison. We show in Fig. $2 \mathrm{a}$ and $2 \mathrm{~b}$ two predictions for the suppression of stronglycoupled bottomonia in an attempt to at least partially map out some of the systematic theoretical uncertainties associated with the use of the AdS/CFT correspondence. Since we used a potential derived in AdS-space dual to maximally supersymmetric Yang-Mills theory, there is no single obvious map between the parameters of QCD and of $\mathscr{N}=4 \mathrm{SYM}$. For the $\alpha_{s}=0.27$ curve, 
we took the relationship between the SYM coupling and temperature and the QCD coupling and temperature to be $\lambda=10$ and $T=T_{Q C D}$. For the $\lambda=5.5$ curve, the coupling constant was set by a comparison to the $q \bar{q}$ potential from lattice and $T=T_{Q C D} / 3^{-1 / 4}$ is a result of assuming the entropies of the QCD and SYM plasmas are the same [16]. We show in Fig. 2a and $2 b$ three predictions for weakly-coupled bottomonia: 1) the suppression using the binding energies we compute from the potential in [14] run through our medium background, 2) the suppression using the binding energies computed in [14] run through our medium background, and 3) the suppression quoted in [13] in which they run the binding energies computed in [14] through their background.

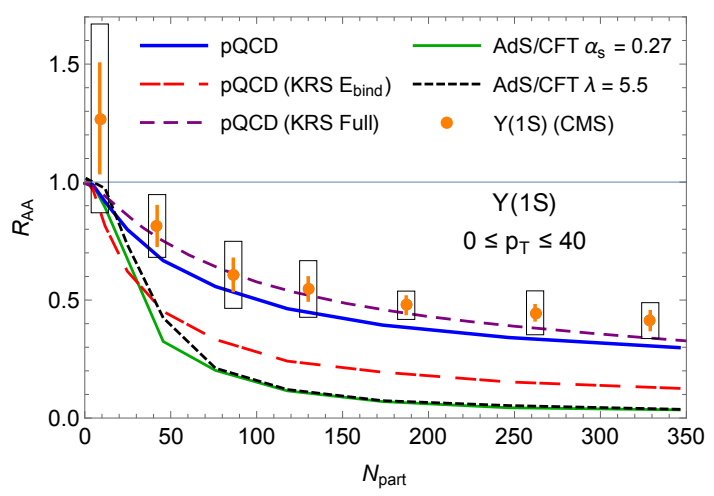

(a)

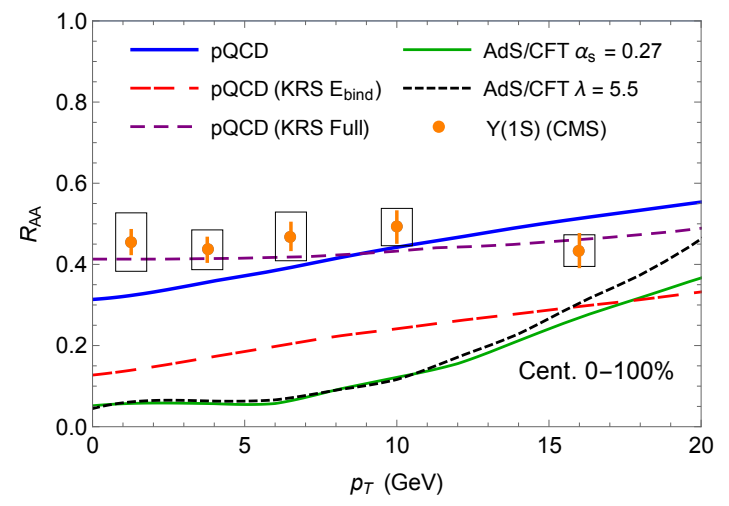

(b)

Figure 2: (a) Nuclear modification factor $R_{A A}$ as a function of the number of participating nucleons $N_{\text {part }}$ for $0 \leq p_{T} \leq 40 \mathrm{GeV}$. (b) Nuclear modification factor $R_{A A}$ as a function of transverse momentum $p_{T}$ for combined centrality classes. In both plots, the thick solid blue line gives our results for weakly-coupled $\Upsilon(1 \mathrm{~S})$, and the dashed-dotted red line that calculated for the binding energy from KRS [14] using our suppression model. The $R_{A A}$ presented in KRS [13] as calculated using their suppression model is given in dashed purple. The solid green and dotted black lines give the results for strongly-coupled $\Upsilon(1 S)$ with coupling constants $\alpha_{s}=0.27$ (and $T_{S Y M}=T_{Q C D}$ ) and $\lambda=5.5$ (and $T_{S Y M}=T_{Q C D} / 3^{1 / 4}$ ), respectively. Data from CMS [17] is included in orange.

\section{Discussion and Outlook}

Our first results for $\Upsilon(1 \mathrm{~S})$ strongly-coupled to a strongly-coupled plasma show binding energies with much larger imaginary parts than those found from the pQCD potential, as well as real parts that become positive within the $T_{c}$ to $3 T_{c}$ range considered. Thus, for the potential models considered here, a strongly-coupled $\Upsilon(1 S)$ interacting with a strongly-coupled plasma melts at a lower temperature than a weakly-coupled $\Upsilon(1 \mathrm{~S})$ interacting with a weakly-coupled plasma. The $\Upsilon(1 S)$ hence appears more strongly bound at weak coupling than at strong coupling, which is surprising. We then input the complex ground-state binding energies that we found using the imaginary time techniques into an implementation of the suppression model described in [13] to determine the $\Upsilon(1 \mathrm{~S})$ nuclear modification factor $R_{A A}$ as a function of the number of participating nucleons, $N_{\text {part }}$, and of transverse momentum, $p_{T}$, respectively. The difference in binding energies for the two coupling scenarios is echoed in the $R_{A A}$ results: from the larger imaginary parts of the strongly-coupled binding energies, we see a significantly larger suppression for strongly-coupled 
$\Upsilon(1 S)$ than for weakly-coupled $\Upsilon(1 S)$. Quantitatively, our full model—comprised of the potential, the resulting quarkonia binding energies, and the translation to $R_{A A}$ - significantly overpredicts the suppression of strongly-coupled bottomonia compared to data. At the same time, our predictions for weakly-coupled bottomonia are consistent with data.

We note that our model for the medium is significantly less sophisticated compared to that used in [13]: our background is an optical Glauber model as opposed to the 3+1D viscous anisotropic hydrodynamics in that work. Our medium incorporates only Bjorken expansion, whereas the background in [13] includes transverse expansion and entropy production. Therefore the plasma in [13] cools faster than ours, leading to our model showing more dissociation for the same binding energies. The extent of the sensitivity of $R_{A A}$ to the background used is surprisingly large. With the only difference being the background geometry used, we ran the binding energies from [14] through our suppression model and found an $R_{A A}$ a factor of two smaller than that shown in [13]. In contrast to the favorable comparison between the pQCD-based results of [13] and the CMS data [17], if we assume our weak coupling binding energies are more accurate than those of [14], then computing $R_{A A}$ with the more sophisticated background from [13] would likely yield a significant underprediction of the suppression of bottomonia. At strong coupling, with a potential derived from AdS/CFT as described in [6], it seems unlikely that the use of a more sophisticated background would reduce the suppression of bottomonia enough that the predicted $R_{A A}$ would be consistent with data; however, the differences from using a more sophisticated background, suppression model, and velocity dependent potential may ultimately be sufficient for future strongly-coupled quarkonia predictions to be consistent with current data. We note that our suppression calculations do not consider feed-down from higher excited states $\Upsilon(\mathrm{nS}), n>1$ for either the pQCD or AdS/CFT potentials. Considering feed-down, however, would only serve to suppress the $R_{A A}$ further. Hence, our qualitative conclusions about strongly-coupled quarkonia would remain unchanged should higher excited states be included.

\section{Acknowledgments}

Support from the South African National Research Foundation and the SA-CERN Consortium are gratefully acknowledged.

\section{References}

[1] T. Matsui and H. Satz, Phys. Lett. B178 (1986) 416.

[2] A. Beraudo, J. P. Blaizot and C. Ratti, Nucl. Phys. A806 (2008) 312 [0712.4394].

[3] A. Rothkopf, T. Hatsuda and S. Sasaki, Phys. Rev. Lett. 108 (2012) 162001 [1108 . 1579].

[4] S.-J. Rey, S. Theisen and J.-T. Yee, Nucl. Phys. B527 (1998) 171 [hep-th/ 9803135$].$

[5] A. Brandhuber, N. Itzhaki, J. Sonnenschein and S. Yankielowicz, Phys. Lett. B434 (1998) 36 [hep-th/9803137].

[6] J. L. Albacete, Y. V. Kovchegov and A. Taliotis, Phys. Rev. D78 (2008) 115007 [0 807 . 4747].

[7] H. Liu, K. Rajagopal and U. A. Wiedemann, Phys. Rev. Lett. 98 (2007) 182301 [hep-ph/0607062]. 
[8] W. A. Horowitz, Nucl. Phys. A904-905 (2013) 186c [1210.8330].

[9] R. Morad and W. A. Horowitz, JHEP 11 (2014) 017 [1 409 . 754 5].

[10] R. Hambrock and W. A. Horowitz, in 8th International Conference on Hard and Electromagnetic Probes of High-energy Nuclear Collisions: Hard Probes 2016 (HP2016) Wuhan, Hubei, China, September 23-27, 2016, 2017, 1703.05845 , https://inspirehep.net/record/1518153/files/arXiv:1703.05845.pdf.

[11] A. Andronic et al., Eur. Phys. J. C76 (2016) 107 [1506.03981].

[12] G. Aarts et al., Eur. Phys. J. A53 (2017) 93 [1612.08032].

[13] B. Krouppa, R. Ryblewski and M. Strickland, Phys. Rev. C92 (2015) 061901 [1507. 03951 ].

[14] M. Margotta, K. McCarty, C. McGahan, M. Strickland and D. Yager-Elorriaga, Phys. Rev. D83 (2011) 105019 [1101.4651].

[15] N. N. Barnard and W. A. Horowitz, 1706.09217.

[16] S. S. Gubser, Nucl. Phys. B790 (2008) 175 [hep-th/ 0612143$].$

[17] CMS collaboration, V. Khachatryan et al., Phys. Lett. B770 (2017) 357 [1611. 01510 ]. 